\title{
Balkanologie
}

Balkanologie Revue d'études pluridisciplinaires

Vol. X, $n^{\circ} 1-2 \mid 2008$

Volume $X$ Numéro 1-2

\section{Balkans-Caucase : introduction au dossier}

Taline Ter Minassian

\section{(2) OpenEdition}

Journals

Édition électronique

URL : https://journals.openedition.org/balkanologie/361

DOI : 10.4000/balkanologie.361

ISSN : 1965-0582

\section{Éditeur}

Association française d'études sur les Balkans (Afebalk)

\section{Référence électronique}

Taline Ter Minassian, « Balkans-Caucase : introduction au dossier », Balkanologie [En ligne], Vol. X, n 1-2 | 2008, mis en ligne le 03 juin 2008, consulté le 08 décembre 2022. URL : http://

journals.openedition.org/balkanologie/361 ; DOI : https://doi.org/10.4000/balkanologie.361

Ce document a été généré automatiquement le 17 décembre 2020.

Tous droits réservés 


\title{
Balkans-Caucase : introduction au dossier
}

\author{
Taline Ter Minassian
}

1 Ce n'est pas la première fois que Balkanologie porte son regard sur les questions caucasiennes: en juillet 1997 la toute première livraison de la revue proposait un article d'Ivan Ivekovic, professeur de science politique à l'AUC (American University in Cairo) sur "Les laboratoires yougoslave et caucasien: Etat, Développement et réajustement global dans l'après-guerre froide ». Dix ans plus tard, l'idée d'un dossier tentant de mettre en parallèle les Balkans et le Caucase, arrive à point nommé pour inaugurer le nouveau format de la revue, désormais en ligne. Ce dossier à vrai dire ne fait qu'ouvrir une voie : la parenté entre le monde balkanique et caucasien au sens large semble évidente pour qui s'intéresse en même temps à l'histoire de l'Empire ottoman et de l'Empire russe. Terres montagneuses où les "valeurs des hautes terres $\rrbracket^{1}$ ont participé à l'élaboration des sentiments nationaux, la Péninsule balkanique et l'Isthme transcaucasien entretiennent une relation de similitude géographique et historique que semble démentir à première vue l'ouverture méditerranéenne de l'une, l'absence d'insularité de l'autre mais qu'affirme au contraire une commune complexité géomorphologique issue d'une situation de contact entre plaques tectoniques. Terres de marges impériales, l'histoire des Balkans et du Caucase, qui intéresse à l'époque contemporaine l'Empire austro-hongrois, l'Empire ottoman et l'Empire russe a généré des situations comparables de mosaïque ethno-religieuse où l'émergence des sentiments nationaux a poursuivi un schéma souvent analogue, identifié par le célèbre ouvrage de Miroslav $\mathrm{Hroch}^{2}$. On n'en finirait pas de dresser l'inventaire des similitudes entre ces deux espaces, de la musique à l'humour en passant par la nourriture. Ajoutons à cela un commun héritage de quelques décennies de régime communiste façonnant plus ou moins les mentalités, les modes de vie et les paysages urbains très comparables, restitués dans les œuvres cinématographiques comme par exemple, 12 h08 à l'Est de Bucarest de Corneliu Porumboiu ou Le voyage en Arménie de Robert Gediguian. Mais ce dossier entend suggérer aussi qu'au-delà des parentés et des similarités, les interactions entre Balkans et Caucase, demeurées jusqu'ici un terrain largement délaissé tant par les spécialistes des Balkans que par ceux du Caucase et de la 
Transcaucasie, pourraient stimuler un certain renouvellement historiographique. Notre dossier comporte trois contributions qui ouvrent de ce point de vue des perspectives non pas simplement comparatives mais «interactives» de l'histoire des Balkans et du Caucase. Sans aucune concertation préalable de leurs auteurs, les trois contributions présentées ont pour épicentre Constantinople-Istanbul, l'ancienne capitale ottomane jouant encore aujourd'hui largement son rôle de pont entre Orient et Occident. L'article de Pierre Voillery consacré à la perception de la résistance tchétchène et à son héros historique -le célèbre Imam Chamyl- dans le premier journal bulgare de Constantinople en 1854-1859 , le Carigradski Vestnik met en évidence, l'existence -même limitée- d'une "perception" des problèmes caucasiens dans ce journal dont le rôle a été si important dans la formation de l'identité nationale bulgare au xIX siècle. Consacré à une œuvre "d'histoire immédiate » des guerres balkaniques de 1912-1913, l'article d'Anahide Ter Minassian propose une analyse et une mise en perspective de l'ouvrage d'Aram Andonian, Histoire générale illustrée de la guerre balkanique, publié en arménien en août 1913 à Constantinople. Elle démontre par là comment l'ouvrage s'insère dans la propagande russe lors de cet ultime épisode de la Question d'Orient dont la conclusion -pertes des provinces balkaniques de l'Empire ottoman, «triomphe » des identités nationales- ne peut qu'inspirer les autres minorités de l'Empire ottoman, et les Arméniens en premier lieu. A plus d'un siècle de distance, la contribution de Jean-François Pérouse envisage le rôle d'Istanbul dans le système international des mobilités entre Transcaucasie et Balkans. Il montre comment Istanbul est redevenue après la chute de l'Union Soviétique une métropole attractive drainant des flux migratoires provenant d'une vaste région s'étendant des Balkans au Caucase via la Transcaucasie.

2 Notre petit dossier s'intègre cependant dans un cadre problématique plus large mêlant l'histoire des Balkans et du Caucase en envisageant tout autant les similarités que les interactions. Et tout d'abord, les luttes révolutionnaires balkaniques n'ont elles pas fourni au xIx ${ }^{\mathrm{e}}$ siècle un modèle d'action aux autres minorités de l'Empire ottoman ? Aux yeux des révolutionnaires arméniens, les Balkans ont été en effet fixé le paradigme de l'action révolutionnaire dans l'Empire ottoman. Les épisodes successifs de la grave crise de 1876-1878, les révoltes de Bosnie et d'Herzégovine, la répression de l'insurrection bulgare, la guerre russo-turque et enfin le traité de San Stefano (3 mars 1878) ont enseigné que l'intervention des puissances en faveur des nations balkaniques avait été fondamentale. Et c'est d'ailleurs dans l'article 16 du traité de San Stefano dont les termes -posant le principe de réformes immédiates en faveur des Arméniens de l'Empire ottoman- ont été négociés au cours des démarches entreprises par le patriarche Nerses Varjabedian auprès des diplomates russes que l'Arménie, mentionnée pour la première fois, a fait son entrée sur la scène internationale. Quelques mois plus tard, le traité de Berlin (juillet 1878) qui réglait essentiellement les questions balkaniques, réduisant notamment à néant le rêve d'une Grande Bulgarie, réitérait dans son article 61 des promesses de réforme en faveur des Arméniens de l'Empire ottoman tout en les soumettant à un improbable contrôle des puissances. La stratégie de l'internationalisation de la question arménienne a ainsi découlé de l'observation directe des différentes phases des mouvements nationaux balkaniques. "Comme les 'hentchak', les 'dachnak' espèrent l'intervention des puissances en faveur du mouvement arménien, car ils ont observé que, dans l'Empire ottoman, la liberté des peuples balkaniques n'a pas été acquise par les seuls actes des 'haïdouk' (" partisans») grecs, serbes ou bulgares, dont l'héroïsme seul ne pouvait suffire devant l'armée ottomane, mais qu'elle résulte de 
l'intervention européenne ». Ces "haïdouk» bulgares dont le mode d'action inspire ces primitifs de la révolte que sont les "fedaïs » arméniens, révolutionnaires armés -mais aussi héritiers $\mathrm{du}$ banditisme rural- voués à l'autodéfense de la paysannerie arménienne des vilayets orientaux de l'Empire ottoman. Parmi toutes les nations balkaniques, la Bulgarie est une source d'inspiration. "Il est incontestable que l'émancipation de la Bulgarie a inspiré la tactique des révolutionnaires arméniens, qui analysent jusqu'à satiété l'enchaînement de ses différentes phases: mouvement haïdouk, insurrection populaire, 'atrocités bulgares', interpellations parlementaires, intervention russe, autonomie de la Bulgarie. Ils tentent de transplanter ce modèle, où sont concentrées toutes les expériences balkaniques, en Asie Mineure, dans une région multinationale où les Arméniens sont minoritaires, ce qui exclut la phase capitale de l'insurrection populaire et condamne le mouvement arménien à se développer comme un mouvement de minorité chrétienne butant sur l'incompréhension et l'hostilité des masses musulmanes. Enfin, au début des années 1890, date à laquelle la subversion arménienne se développe, le modèle bulgare est devenu caduc: l'intervention de la Russie, qui se tourne résolument vers l'Extrême-Orient et qui, loin de soutenir les révolutionnaires arméniens, les pourchasse, est improbable $"^{4}$. On verra justement comment la publication du livre d'Aram Andonian sur les guerres balkaniques s'intègre en 1912 dans le contexte de la relance de la question arménienne sous l'égide de la diplomatie russe. Cette dernière constitue incontestablement le commun dénominateur des questions balkaniques et caucasiennes au XIX ${ }^{\mathrm{e}}$ siècle que l'on peut envisager comme les deux versants de la Question d'Orient. Récemment, Balkanologie a reçu l'ouvrage d'Alexander Bitis consacré à la Russie et à la Question d'Orient ${ }^{5}$, vieux sujet renouvelé ici par l'apport de sources d'archives -en premier lieu les archives militaires de Russie, RGVIA à Moscou- demeurées longtemps inaccessibles aux chercheurs occidentaux. Ce livre étudie dans un même mouvement l'expansion militaire et diplomatique de la Russie aux dépens de l'Empire ottoman et de la Perse pendant le premier tiers du $\mathrm{XIX}^{\mathrm{e}}$ siècle et traite sur le même plan des Balkans, du Caucase et de la Transcaucasie. C'est dire si la publication de cet ouvrage tombe à point nommé car jusqu'ici l'historiographie occidentale était largement fondée sur une perception européocentriste de la Question d'Orient, séparant du même coup la compréhension des événements balkaniques et caucasiens. Or, les Balkans et la Transcaucasie, bien qu'éloignés géographiquement, forment un seul tenant dans la logique de l'expansion militaire russe qui par exemple au moment du traité d'Andrinople (1829) prend en tenailles les deux rives de la Mer Noire avançant la frontière russe en Arménie et en Géorgie tout en procédant à l'occupation des principautés danubiennes de Moldavie et de Valachie. L'imposante somme d'Alexander Bitis envisage dans un certain désordre, après plusieurs chapitres préparatoires consacrés à l'organisation et à la doctrine de la diplomatie et de l'armée impériale russe, le rôle de la Deuxième Armée dans la révolution grecque en 1821-1822, la guerre russo-perse (1826-1828), la guerre russoturque (1828-1829) et l'utilisation dans ce contexte des irréguliers balkaniques, le traité d'Andrinople et ses conséquences internationales et l'occupation des principautés danubiennes (1828-1834). Mais plus que la chronologie de cette phase de la Question d'Orient, c'est surtout l'étude des structures politiques et militaires russes, de la propagande d'Etat et de l'opinion publique qui constituent l'apport de l'ouvrage d'Alexander Bitis.

3 Le lecteur de ce dossier pourra se convaincre de la nécessité d'aborder en même temps les Balkans et le Caucase s'agissant de la Question d'Orient et tout l'intérêt qu'il y aurait aussi à promouvoir des études sur les perceptions réciproques de ces deux espaces. Car 
s'il y eut incontestablement une perception caucasienne des problèmes balkaniques, existe-t-il en retour une perception balkanique des problèmes caucasiens? Difficile question à laquelle peu de spécialistes des Balkans ont voulu répondre, tout au moins dans le cadre de ce numéro, à la notable exception de Pierre Voillery. Il est vrai que la démarche proposée, celle du comparatisme et des regards croisés, suppose de la part des spécialistes des aires régionales, des Balkans comme du Caucase, un effort pluridisciplinaire souvent dédaigné au nom d'un principe de "crédibilité scientifique ». D'autre part, peu d'initiatives à ce jour ont amené à se faire rencontrer les spécialistes de l'une ou l'autre zone. Et il est d'ailleurs significatif que l'étude des diasporas ait été jusqu'à présent le seul terrain de rencontre notable. Il faut saluer ici la publication des Actes d'un colloque européen et international réuni à l'Ecole française d'Athènes (4-7 octobre 2001) rassemblant des contributions sur les Arméniens et les Grecs en diaspora ${ }^{6}$ ainsi qu'une cartographie comparative de ces deux diasporas. Volumineux ouvrage qui pose le cadre historique et géographique, met en perspective dans l'espace et dans le temps les territoires de ces deux diasporas, pose la question de la relation entre l'Etat national et la diaspora et envisage enfin la formation du lien social en diaspora. À l'origine de cette initiative, le géographe Michel Bruneau, spécialiste des Grecs pontiques, une spécialité qui sans doute le prédisposait à faire le lien entre des spécialités souvent isolées. "La diaspora grecque peut ainsi être vue comme un vaste réseau dans lequel les populations circulent, se dispersent, se recomposent, se redistribuent sans cesse. L'identité portée par une langue, une religion et une iconographie constitue le lien essentiel entre tous ces éléments dispersés, qui sont en évolution et en mouvement perpétuel. C'est ce noyau identitaire, relevant de la longue durée, qui empêche la dissolution du tout par assimilation dans les pays d'accueil, comme on le constate chez les peuples-monde que sont les Grecs ou les Arméniens. La Méditerranée orientale et le Proche-Orient d'abord, l'ensemble du bassin méditerranéen, surtout ses côtes européennes, la péninsule des Balkans ensuite, l'Europe centrale et orientale, enfin l'Europe occidentale ont été successivement les espaces où se sont déployés les réseaux de la diaspora grecque jusqu'à la fin du XIX siècle (...). Les flux centrifuges et centripètes des populations grecques au sein de ces espaces réticulés ont souvent été causés par des désastres et des massacres récurrents dans l'histoire grecque comme dans celle de la diaspora, mais aussi par le choix des migrants eux-mêmes en fonction de difficultés économiques et parfois politiques. Le territoire de l'Etat-nation est autant, sinon davantage, l'œeuvre des populations de la diaspora "rapatriées " et réfugiées que des autochtones "7. L'étude des diasporas permet ainsi d'étendre le champ des regards croisés entre Balkans et Caucase et permet d'observer comme le fait par exemple Tamara Galkina, géographe rattachée à l'Académie des Sciences de Moscou, les relations entre les diasporas grecque et arménienne dans la province de Stavropol, c'est-à-dire dans la région du piémont caucasien et d'envisager des études en miroir sur les Arméniens en Grèce ${ }^{8}$ ou sur les Grecs en Arménie ${ }^{9}$. Naturellement, il conviendrait de poursuivre sans pesanteur ni systématisme de telles études concernant les diasporas caucasiennes dans les pays de la Péninsule balkanique et inversement sur les populations d'origine balkanique dans la région caucasienne et transcaucasienne. Si cette approche nous ramène à la question des mobilités et des flux migratoires entre Transcaucasie et Balkans, étudiés par JeanFrançois Perouse à Istanbul, elle attire aussi l'attention sur l'histoire récente et moins récente de ces petites communautés dispersées en Bulgarie ou en Serbie. Babken Simonian ${ }^{10}$, poète, écrivain, traducteur et publiciste, spécialiste des relations culturelles et littéraires arméno-serbes s'intéresse justement depuis des années à l'histoire de la colonie arménienne de Serbie dont les origines pourraient remonter au XIII ${ }^{e}$ siècle. Le 
voyage de Saint Sava en Arménie cilicienne en 1235 marquerait le point de départ des relations arméno-serbes mais la colonie arménienne de Belgrade remonte au début du $\mathrm{XVII}_{\mathrm{e}}$ siècle : la plupart des migrants étaient originaires du Nakhitchevan, d'Erzeroum, de Erevan et même de Crimée où sévissaient sécheresse et famine. L'église arménienne de Belgrade date de la seconde moitié du XviI $e$ siècle tandis que celle de Novi Sad, démolie en 1963, avait été érigée en 1746. S'il ne reste plus aujourd'hui qu'une colonie arménienne de 200 personnes en Serbie -dont 150 à Belgrade- la diaspora arménienne en Serbie nourrie de nouveaux apports de réfugiés pendant les années 1920, mérite certainement une étude approfondie tout comme les autres communautés dispersées dans les Etats de la Péninsule balkanique.

4 Les Balkans sont enfin un outil de référence pour penser et enseigner l'histoire et la géopolitique du Caucase, de la Transcaucasie et même du Moyen-Orient, permettant dans une certaine mesure une tentative de modélisation des conflits territoriaux. Ainsi, on peut distinguer le processus de «balkanisation»-processus de morcellement d'entités impériales comme l'Empire ottoman ou l'Empire habsbourgeois en Etats nationaux antagonistes- de celui de la « libanisation »-déliquescence interne d'un Etat multiconfessionnel, ordre milicien, occupation d'Etats voisins- et même inventer de nouveaux concepts comme celui de la «karabaghisation » qui décrit la situation d'un Etat dont les institutions sont noyautées par les représentants d'une "exclave " détachée d'un Etat voisin. Là encore, les Balkans tout comme l'espace caucasien et transcaucasien offre un terrain intéressant de comparaison à travers la question des «quasi-Etats» tels le Kosovo, la Transnistrie, l'Abkhazie, l'Ossétie du sud, le HautKarabagh, Chypre du nord. Ces entités autonomes s'imposent aujourd'hui comme un enjeu politique central dans la consolidation des Etats que ceux-ci soient issus de l'ancien Empire soviétique ou de l'ex-Yougoslavie. Ces quasi-Etats que la science politique distingue des Etats faibles (weak states) du fait des tensions ethniques, religieuses, linguistiques et de leur désorganisation économique tels la BosnieHerzégovine et la Macédoine, se distinguent également des Etats défaillants (failed states) dont la fonction étatique est mise en faillite par une guerre permanente, des collapsed states aux mains des mafias et de guerillas ainsi que des micro-Etats. A l'exception de la Transnistrie ${ }^{11}$, les quasi-États dans l'espace post-soviétique sont d'anciennes entités autonomes ayant déclaré leur souveraineté au moment de la chute de l'Union Soviétique: ce sont des Etats indépendants de facto car ils ne sont pas reconnus en droit international. Pourtant les quasi-Etats jouent bien évidemment un rôle -et même souvent un rôle de premier plan - sur la scène internationale. Dans l'espace post-soviétique, ils sont l'un des terrains où s'exerce la politique d'influence de la Russie dans son «étranger proche » tout comme le Kosovo est le terrain d'action de l'Alliance occidentale. Certains commentateurs américains constatent que «le Caucase est malheureusement devenu une nouvelle incarnation des Balkans, une région oubliée d'Europe où un vieil Empire hostile s'en prend à des peuples plus faibles que lui et où des guerres risquent à tout moment d'éclater $~^{12}$, mais c'est surtout la perspective d'une indépendance du Kosovo qui pourrait à l'avenir instaurer un nouveau jeu de miroirs entre Balkans et Caucase. Après le Kosovo, l'Abkhazie ou le Haut-Karabagh pourraient eux aussi prétendre à la remise en cause du principe de l'intangibilité des frontières en Europe (Accords d'Helsinki, 1975) au nom du droit des peuples à disposer d'eux-mêmes. En tout cas les quasi-Etats balkaniques tout comme les quasi-Etats eurasiens sont désormais au centre du nouveau «Grand Jeu » d'influence entre la Russie et le camp occidental. En janvier 2006, Vladimir Poutine n'a-t-il pas déclaré que «si le principe de l'indépendance du 
Kosovo venait à être reconnu, plus aucun obstacle n'existerait alors pour reconnaître le droit de la Transnistrie, de l'Abkhazie et de l'Ossétie du Sud à réaliser leur droit à l'autodétermination ». Ainsi, au XxI ${ }^{\mathrm{e}}$ siècle, la «nouvelle » Question d'Orient mêle toujours aussi étroitement les enjeux balkaniques et les enjeux caucasiens.

\section{NOTES}

1. Brunnbauer (Ulf), Pichler (Robert), «Mountains as 'lieux de mémoire', Highland values and nation-building in the Balkans », in Balkanologie, $\mathrm{n}^{\circ} 1-2$, décembre 2002, pp. 77-100.

2. Hroch (Miroslav), Social preconditions of national revival in Europe. A comparative analysis of the social composition of patriotic groups among the smaller European nations, Cambridge, Cambridge University Press, 1985.

3. Ter Minassian (Anahide), «L'Arménie et l'éveil des nationalités (1800-1914) », in Histoire du Peuple Arménien, sous la direction de Gérard Dedeyan, Toulouse, Privat, réédition 2007, p. 505. Voir également du même auteur, La Question Arménienne, Marseille, Parenthèses, 1983 et Histoires Croisées, Arménie 1890-1900, Marseille, Parenthèses, 1997.

4. Ibid, p. 508.

5. Bitis (Alexander), Russia and the Eastern Question, Army, Government and Society, 1815-1833, Oxford, Oxford University Press, 2006.

6. Bruneau (Michel), Hassiotis (Ioannis), Hovanessian (Martine), Mouradian (claire) (éd.), Arméniens et Grecs en diaspora: approches comparatives, Actes du colloque européen et international organisé à l'Ecole française d'Athènes (4-7 octobre 2001), Athènes, Ecole française d'Athènes, Champs helléniques et contemporains, 2007.

7. Bruneau (Michel), «Comment définir la diaspora grecque ? ", in Arméniens et Grecs en diaspora : approches comparatives, op.cit., p. 28.

8. Antoniou (Panayota), "L'intégration des Arméniens en Grèce, La constitution passée et présente d'une partie de la diaspora arménienne », ibid., pp. 385-396.

9. Charatsidis (Elevtheris), « Particularités ethnosociales de la population grecque des villes de l'Arménie orientale et de la région de Kars », ibid., pp. 397-408.

10. Simonian (Babken), « Armjane v Serbii v XIII-XX vekakh» in Aniv, n³ (6), 2006, pp. 14-24.

11. Parmentier (Florent), «La Transnistrie, Politique de légitimité d'un Etat de facto », in Le Courrier des Pays de l'Est, n¹061, mai-juin 2007, pp.69-75.

12. Jackson (Bruce P.), The Weekly Standard, Washington, repris par Courrier International, $\mathrm{n}^{\circ} 846$, 18-24 janvier 2007, p. 35. Bruce P.Jackson dirige l'association néo-conservatrice Project on Transitional Democracies agissant dans les pays de l'ancien bloc soviétique. 
INDEX

Index géographique : Balkans, Caucase, Transcaucasie, Istanbul

Mots-clés : question d'Orient, nationalismes, diasporas, migrations

\section{AUTEUR}

TALINE TER MINASSIAN

Maître de conférences en histoire contemporaineUniversité Jean Monnet (Saint-Etienne) 